\title{
Estructura de los bosques de Gleditsia triacanthos en función de la edad (valle de La Sala, Tucumán, Argentina)
}

\author{
Sergio J. Ceballos ${ }^{\varpi ;}$; Yohana G. Jimenez \& Romina D. Fernandez
}

Instituto de Ecología Regional (CONICET-UNT).

\begin{abstract}
Resumen. Gleditsia triacanthos es una especie exótica invasora, ampliamente distribuida en varias ecorregiones de la Argentina. Cuando invade, esta especie forma bosques monoespecíficos de estructura y dinámica poco conocida. Investigar estos aspectos es fundamental para evaluar el comportamiento de la invasora y la capacidad de las especies nativas para colonizar los bosques invadidos. En este trabajo describimos la estructura y la composición, e inferimos la dinámica temporal de los bosques de G. triacanthos en el valle de La Sala, el principal foco de invasión de la especie en la provincia de Tucumán. En 2019 establecimos parcelas en cuatro parches de bosques dominados por G. triacanthos, de 9, 18, 25-29 y 36-37 años de edad. Allí registramos la densidad, la riqueza y la altura de árboles $\geq 5 \mathrm{~cm}$ de diámetro, los renovales y otras características como el estado de los árboles, presencia de ganado y claros. El bosque de 9 años tenía baja altura y baja densidad, con presencia de claros aún no colonizados por G. triacanthos. El bosque de 18 años fue más denso que el resto. Los árboles aumentaron su altura y su diámetro hacia los bosques de 25-29 y 36-37 años, y también aumentaron el número de árboles caídos o inclinados. Suponemos que G. triacanthos elimina por competencia a Vachellia aroma, una especie arbórea nativa que coloniza tempranamente parches invadidos. En etapas tardías, otras especies nativas pudieron establecerse, pero en bajas densidades. Desde su establecimiento hasta los bosques más viejos, los parches de G. triacanthos se mantuvieron casi monoespecíficos debido a la alta densidad de esta invasora y a la facilitación por parte del ganado presente en el valle.
\end{abstract}

[Palabras clave: especies nativas, plantas invasoras, riqueza de especies, Yungas]

\begin{abstract}
Aвstract. Structure related to the age of Gleditsia triacanthos forests in La Sala Valley (Tucuman, Argentina). Gleditsia triacanthos is an exotic invasive species in Argentina, widely distributed in several ecoregions. This species forms monospecific forests whose structure and dynamics remain poorly understood. These aspects are key to assess the behavior of the invader and the capacity of native species to colonize invaded forests. In this study we describe the structure and composition, and infer the temporal dynamics of G. triacanthos forests in La Sala Valley, an area highly invaded by this species in the province of Tucuman. In 2019, in four forest patches of 9, 18, 25-29 and 36-37 years old dominated by G. triacanthos, we established experimental plots and registered density, species richness and height of trees $\geq 5 \mathrm{~cm}$ in diameter, recruits, and other characteristics such as tree health and presence of cattle, gaps or open spaces. The 9-year-old forest had low height, low density and open spaces not yet colonized by G. triacanthos. The 18-year-old forest had higher density than the rest. In older forests (25-29 and 36-37-years-old patches), we detected an increase in the height and diameter of trees, and in the number of fallen and leaning trees. We suppose that Vachellia aroma, a native tree species that colonize early stages of invaded forests, was rapidly outcompeted by G. triacanthos. Lately, other native species colonized forests, but at low densities. G. triacanthos forests were almost monospecific because of its high density and the facilitation effect of the cattle in the valley, which consume and disperse the seeds.
\end{abstract}

[Keywords: native species, invasive plants, species richness, Yungas]

\section{INTRODUCCIÓN}

Una especie exótica e invasora es aquella cuya introducción y expansión por acción humana amenaza la diversidad biológica, la seguridad alimentaria, la salud y el bienestar de las personas (IPBES 2020). Entre estas especies se encuentran árboles y arbustos introducidos en todo el mundo, que se dispersaron desde los sitios donde fueron plantados y que se convirtieron en especies naturalizadas o invasoras (Richardson and Rejmánek 2011). En diferentes ecosistemas, los árboles y los arbustos se encuentran entre las especies invasoras más conspicuas,

Editora asociada: Patricia Kandus dañinas y, en algunos casos, muy estudiadas (e.g., las especies invasoras del género Pinus). Sin embargo, muchas de estas especies se convirtieron en invasoras recientemente o recibieron poca atención, por lo que varios aspectos de su invasión permanecen poco conocidos, tales como la estructura de los bosques invadidos, restauración y cambios composicionales en el largo plazo (Richardson and Rejmánek 2011).

Gleditsia triacanthos, conocida como "acacia negra" o "acacia de las tres espinas" (Fabaceae), es una especie arbórea originaria de Norteamérica, que fue introducida y

Recibido: 29 de Noviembre de 2019

Aceptado: 1 de Abril de 2020 
se convirtió en invasora en varios países de Sudamérica, Oceanía, África y Europa (Csurhes and Kriticos 1994; Zalba and Villamil 2002; Caballero 2013). En la Argentina fue introducida a fines del siglo XXVIII para cercos y como árbol ornamental, y actualmente invade las Pampas, el Chaco Seco, las Yungas y el Espinal (Prieto et al. 2004; Fernández et al. 2017). El ganado favorece su invasión al consumir y dispersar sus vainas, al igual que los disturbios antrópicos que "abren espacios" para su colonización. En comparación con especies arbóreas nativas, G. triacanthos es una invasora exitosa debido a su habilidad competitiva, a su plasticidad fenotípica y a su capacidad de adaptarse a diferentes ambientes (Fernández et al. 2017; Tognetti et al. 2019). Al invadir, cambia la fisonomía y la estructura de las comunidades locales (e.g., convierte pastizales nativos en bosques monoespecíficos), e impacta de forma negativa sobre la flora y la fauna nativas, a la vez que ocasiona cambios en los procesos ecosistémicos (Ghersa and León 2001; Zalba and Villamil 2002).

Varios aspectos de la ecología de la invasión de G. triacanthos se investigaron en la Argentina, como su impacto sobre diferentes componentes del ecosistema, su habilidad competitiva en comparación con otras especies arbóreas nativas y el rol de disturbios antrópicos en su colonización, entre otros (Marco and Páez 2000; Ghersa and León 2001; Di Marzio et al. 2009; Ferreras et al. 2014; Capó et al. 2016). En una revisión sobre la invasión de G. triacanthos se observó que 62 de 91 trabajos publicados sobre esta especie se realizaron en la Argentina (Fernández et al. 2017). Este resultado no es sorprendente debido a que $G$. triacanthos se encuentra entre las exóticas arbóreas más agresivas de la Argentina, donde afecta negativamente la flora y la fauna, invade áreas protegidas y ocasiona problemas económicos en campos agrícolas (Marco and Páez 2000; Ghersa and León 2001; Fernández et al. 2017).

Gleditsia triacanthos forma bosques monoespecíficos o mixtos, cuya estructura y dinámica permanecen poco estudiadas. Se sabe que G. triacanthos es una de las especies dominantes de estos bosques debido a su comportamiento como pionera (i.e., es una de las primeras colonizadoras de áreas abiertas y disturbadas). Al establecerse, forma arbustales bajos y espinosos que crecen y generan bosques con una densidad variable (Faggi et al. 2006; Quiroga and Juliá 2011). Por ejemplo, en un bosque monoespecífico en las Pampas, G. triacanthos puede alcanzar una densidad de 1600 árboles por hectárea (Mazia et al. 2010), y en un bosque mixto en las Yungas puede tener una densidad de 110 árboles por hectárea (Bustos 1991). Sin embargo, en la mayoría de los sitios donde invade no existen datos publicados sobre su densidad, biomasa, área basal u otros aspectos básicos de su estructura y dinámica (Ghersa and León 2001; Giorgis and Tecco 2014; Fernández et al. 2017). Obtener esta información es útil para conocer el funcionamiento de los bosques dominados por G. triacanthos, evaluar estrategias de control, conocer la capacidad de estos bosques para almacenar carbono y la dinámica de colonización de árboles nativos de interés para la restauración de la composición nativa. En particular, sería importante determinar si con el paso del tiempo, los bosques exóticos de G. triacanthos podrían ser reemplazados, sin intervención, por especies nativas, o si es necesario programar un plan de manejo para fomentar dicho reemplazo. El objetivo de este estudio es describir la estructura y la composición de bosques de diferentes edades dominados por G. triacanthos en el valle de La Sala (Tucumán, Argentina). También se discuten algunos aspectos de la dinámica de estos bosques, como cambios estructurales a través del tiempo y la capacidad de colonización de especies de árboles nativos. Debido a diferentes factores (e.g., alta densidad, características intrínsecas de la invasora y dispersión efectiva por el ganado que consume sus vainas), predecimos que la dominancia de $G$. triacanthos se mantiene a través del tiempo en los parches de bosque del valle de La Sala. Debido a la mayor habilidad competitiva de la invasora, se dificultaría la colonización de especies de árboles nativos en estos bosques.

\section{Área de estudio}

Realizamos este estudio en el valle de La Sala, Tucumán $\left(26^{\circ} 45^{\prime} 15^{\prime \prime} \mathrm{S}-65^{\circ} 23^{\prime} 20^{\prime \prime} \mathrm{O}\right)$, ubicado al oeste de la Sierra de San Javier, a aproximadamente $35 \mathrm{~km}$ del Gran San Miguel de Tucumán (GSMT). El valle se encuentra a $1000 \mathrm{~m}$ s.n. m. y presenta un clima subtropical, con un verano cálido y húmedo y un invierno frío y seco. La precipitación media anual es de $1031 \mathrm{~mm}$ y la temperatura media anual es $19.5^{\circ} \mathrm{C}$ (Tognetti et al. 2019). El valle tiene un poblado de 700 habitantes (censo 2010, INDEC), cuya actividad productiva principal es la ganadería y la horticultura. 
Los cambios de uso del suelo fueron transformando el paisaje del valle de La Sala a través del tiempo. Hasta el siglo pasado era un paisaje productivo con campos de maíz, lechuga y ganado. Es posible que como consecuencia de la migración rural-urbana, en particular de los jóvenes, parte de los campos de las zonas más bajas fueron abandonados e invadidos rápidamente por G. triacanthos, a la que se la usaba como cerco y árbol ornamental. Esta colonización convirtió al valle de La Sala en el principal foco de invasión de $G$. triacanthos en la provincia de Tucumán (Grau et al. 2010). Hoy, el paisaje del valle está dominado por campos agrícola-ganaderos y por parches de bosque de $G$. triacanthos rodeados por bosques nativos. La conectividad entre estos parches invadidos varía, y son más extensos y continuos en las zonas de baja pendiente. En el valle, los bosques nativos sobreviven, gracias a la heterogeneidad de la topografía, en las laderas o en las paredes empinadas que delimitan los ríos, y donde el ganado que dispersa a G. triacanthos tiene menos acceso. La composición de estos bosques nativos es típica de las selvas montanas de las Yungas, e incluye también algunos elementos de Chaco Serrano, por lo que se la considera una vegetación de transición (Grau et al. 2010). En ellos se encuentran especies nativas como Juglans australis, Allophylus edulis, y también hay otras exóticas como Morus alba, con menor capacidad invasora en la zona. La fisonomía de los bosques de G. triacanthos es simple, con pastos bajos, poco sotobosque y generalmente un único estrato de árboles. Por su parte, los bosques nativos tienen una fisonomía más compleja, con estrato de dosel, subdosel y sotobosque, presencia de lianas y mayor diversidad. No obstante, los bosques nativos también se encuentran disturbados por la actividad ganadera, al igual que otras fisonomías del valle, como pastizales y arbustales antrópicos (Grau et al. 2010).

\section{Materiales y MÉTOdos}

La caracterización de la estructura de los bosques de G. triacanthos en el valle de La Sala se realizó con el establecimiento de una cronosecuencia de 4 parcelas. Las mismas fueron establecidas en parches de bosques que tenían las siguientes características: dominancia de G. triacanthos, similares niveles de carga ganadera y pendiente, cultivos como uso del suelo previo al establecimiento (e.g., maíz), tamaño igual o mayor a media hectárea y que no se encontraran cercados. Al controlar todos estos factores, asumimos que la dinámica de los parches está controlada por la dominancia de G. triacanthos y por el tiempo. Los parches tenían 9, 18, 25-29 y 36-37 años en 2019, y sus edades fueron determinadas empleando diferentes métodos. Con Google Earth pudimos determinar el momento del abandono del cultivo y, por ende, las edades de los parches de 9 y 18 años. En el caso del parche de 25-29 años, barrenamos 6 árboles y determinamos su edad en el laboratorio contando los anillos de crecimiento con una lupa. Para el parche de 36-37 años utilizamos fotografías aéreas de 1981 e imágenes Landsat 5 de 1984, con resolución espacial de 30×30 $\mathrm{m}$, que permitieron identificar la presencia de cultivos posteriormente reemplazados por un bosque. Esto nos permitió determinar la edad de establecimiento entre ambos años.

Cada parcela es rectangular, tiene un tamaño de $80 \times 60 \mathrm{~m}$ y está dividida internamente en 12 cuadrantes de $20 \times 20 \mathrm{~m}$. La excepción es la parcela de 9 años, cuyas dimensiones son menores debido a que se encuentra en un parche alargado y de menor tamaño (las dimensiones de la parcela son de 40×100 m y está dividida en 10 cuadrantes). Los cuadrantes y los límites de la parcela fueron marcados con estacas de pvc, y dentro de los mismos procedimos a colocar chapas para identificar los árboles con un diámetro a la altura del pecho $\geq 5 \mathrm{~cm}$. Colocamos chapas numeradas a los tallos principales, y chapas con letras A, B y C a los tallos secundarios de cada árbol. Ubicamos las chapas a $1.6 \mathrm{~m}$ desde el suelo y medimos el perímetro de cada tallo a $1.3 \mathrm{~m}$, siguiendo el protocolo de Osinaga Acosta et al. (2014) para el establecimiento de parcelas. También identificamos la especie de cada individuo, estimamos visualmente su altura, anotamos sus características más notables (e.g., inclinado, en mal estado, tronco irregular, etc.), registramos sus coordenadas dentro de la parcela en un sistema X-Y, anotamos la presencia de renovales de árboles nativos y características notables de la parcela (e.g., presencia de tocones). La división de las parcelas en cuadrantes de $20 \times 20 \mathrm{~m}$ y las coordenadas de los árboles con chapas se hicieron con el objetivo de facilitar la medición de los mismos individuos en muestreos que se realizarán en los próximos años. Con la información obtenida de las parcelas realizamos una caracterización de los parches de bosques de $G$. triacanthos considerando las siguientes variables: densidades (de individuos y tallos arbóreos), riqueza de 
especies de árboles (adultos y renovales), tamaño de los árboles (diámetros y alturas) y otras observaciones (e.g., presencia de claros, tocones, rebrotes, estado de los árboles). Con los datos de la cronosecuencia, reemplazando espacio (parcelas de diferentes edades) por tiempo, discutimos la dinámica temporal de los bosques de G. triacanthos.

Analizamos cambios en la estructura del bosque con la edad utilizando modelos lineales generalizados mixtos (GLMM) con las librerías lme y lmerTest en $\mathrm{R}$ ( $\mathrm{R}$ Core Team 2020). Las variables analizadas fueron densidad de tallos, DAP promedio y altura promedio del bosque, considerando los cuadrantes de las parcelas como pseudorepeticiones. Para cada modelo se utilizó la variable como efecto fijo, el cuadrante como efecto aleatorio y una distribución binomial negativa para los errores. Se realizaron pruebas de Tukey a posteriori para comparar las variables estructurales entre las parcelas de diferente edad utilizando la librería emmeans en $\mathrm{R}$.

\section{Resultados}

\section{Bosque de 9 años}

Este bosque se caracterizó por su baja altura (6 m) y baja densidad, con la presencia de espacios abiertos o claros (alrededor del 30\% de la parcela) que aún no fueron colonizados (Tabla 1; Figuras 1 y 2). La densidad de tallos, el DAP y la altura media de los árboles fue significativamente menor en este bosque en comparación con los bosques más viejos (Tabla 2). Registramos 395 tallos / ha de G. triacanthos y sólo 2 tallos/ha de Vachellia aroma (una especie arbórea nativa), por lo que puede ser considerado monoespecífico. Observamos que los árboles jóvenes de G. triacanthos tenían una alta densidad de espinas en el tallo principal y rebrotes secundarios alrededor de su base.

\section{Bosque de 18 años}

Este bosque tenía una alta densidad de G. triacanthos, que superaba los 1000 tallos por hectárea (extrapolación de la densidad de la parcela de 18 años) (Tabla 1; Figura 2). La densidad de tallos en este bosque fue significativamente más alta que la de los bosques de 9 y 25-29 años (Tabla 2). Vachellia aroma también tuvo una densidad más alta que en otros bosques, y muchos de sus tallos se encontraban secos, en mal estado y con algunos rebrotes. Se encontraron renovales de otras especies arbóreas protegidos por las espinas de G. triacanthos, aunque con muy baja densidad. Por hectárea, se registraron 6 renovales de Allophylus edulis, 6 de Xylosma pubescens, 4 de Blepharocalyx salicifolius y 2 de la exótica Morus alba.

\section{Bosque de 25-29 años}

Los árboles de G. triacanthos en este bosque tenían mayor altura y diámetro que en los bosques más jóvenes (Figuras 1 y 2; Tabla 2). $\mathrm{Su}$ densidad fue de 760 tallos por hectárea. Las densidades de G. triacanthos y $V$. aroma fueron más bajas que en el bosque de 18

Tabla 1. Características de las parcelas establecidas en bosques de G. triacanthos en el valle de La Sala, Tucumán.

Table 1. Description of the plots established in G. triacanthos forests in La Sala valley (Tucumán).

\begin{tabular}{|c|c|c|c|c|}
\hline & $\begin{array}{l}\text { Parcela de } \\
9 \text { años }\end{array}$ & $\begin{array}{l}\text { Parcela de } \\
18 \text { años }\end{array}$ & $\begin{array}{l}\text { Parcela de } \\
25-29 \text { años }\end{array}$ & $\begin{array}{l}\text { Parcela de } \\
\text { 36-37 años }\end{array}$ \\
\hline Año de establecimiento & 2010 & 2001 & 1990-1994 & \multirow{2}{*}{$\begin{array}{l}\text { 1982-1983 } \\
\text { Imágenes Landsat y } \\
\text { fotografías aéreas }\end{array}$} \\
\hline Método para definir el establecimiento & Google Earth & Google Earth & Dendrocronología & \\
\hline Tamaño de la parcela (ha) & 0.4 & 0.48 & 0.48 & 0.48 \\
\hline Número de cuadrantes de 20×20 m & 10 & 12 & 12 & 12 \\
\hline $\begin{array}{l}\text { Proporción de G. triacanthos con respecto a } \\
\text { otras especies (\%) }\end{array}$ & 99 & 88 & 99 & 97 \\
\hline Densidad de tallos/ha & 395 & 1075 & 760 & 783 \\
\hline Densidad de individuos/ha & 322 & 848 & 658 & 750 \\
\hline Altura media de los árboles (m) & 6.1 & 8.4 & 10.7 & 9.9 \\
\hline Diámetro medio de los árboles $(\mathrm{cm})$ & 9.9 & 12.1 & 17.1 & 16.4 \\
\hline Riqueza de especies de árboles con $\mathrm{DAP} \geq 5 \mathrm{~cm}$ & 2 & 2 & 4 & 8 \\
\hline Riqueza de renovales de árboles & 2 & 5 & 1 & 6 \\
\hline
\end{tabular}



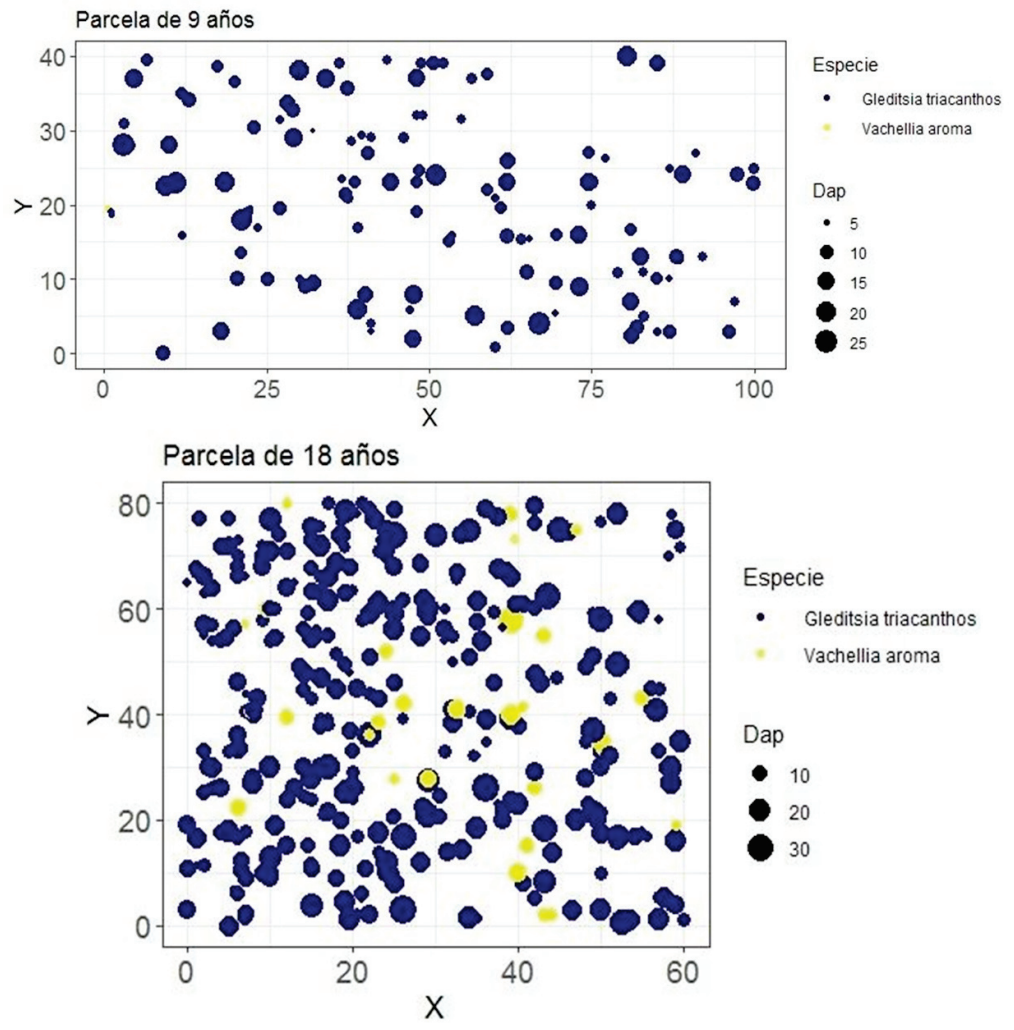

Especie

- Gleditsia triacanthos Vachellia aroma

Dap

- 10

20

30

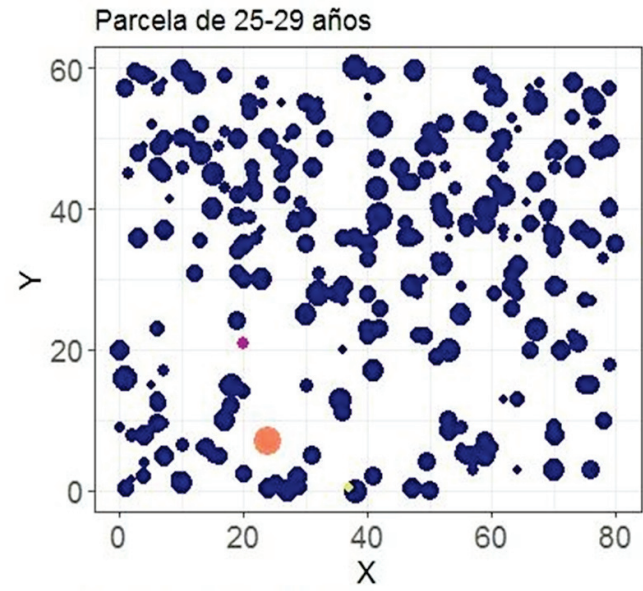

Especie

- Gleditsia triacanthos

- Vachellia aroma

- Morus alba

Xylosma pubescens

Dap

- 10

- 20

30

40

50

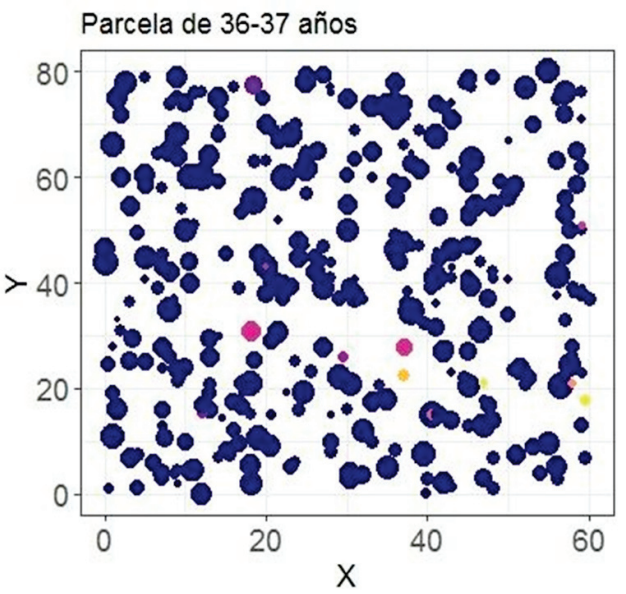

Especie

- Gleditsia triacanthos

- Morus alba

- Xylosma pubescens

- Allophylus edulis

- Azara salicifolia

Celtis ehrenbergiana

Cestrum lorentzianum

Condalia buxifolia

Dap

- 10

- 20

30

40
Figura 1. Disposición espacial de los individuos arbóreos en las parcelas de 9, 18, 25-29 y 3637 años establecidas en parches dominados por G. triacanthos en el valle de La Sala (Tucumán). Cada círculo representa un individuo, el color es la especie a la que pertenece y el tamaño es el DAP (diámetro a la altura del pecho). Los números de los ejes indican el largo y ancho de las parcelas en metros.

Figure 1. Spatial distribution of individual trees in the plots of 9, 18, 25-26, and 36-37 years established in forest patches dominated by G. triacanthos in La Sala valley (Tucumán). Circles represent individual trees, the color is the tree species, and their size is the DBH (diameter at breast height). Axes numbers indicate the dimensions of the plots in meters. 

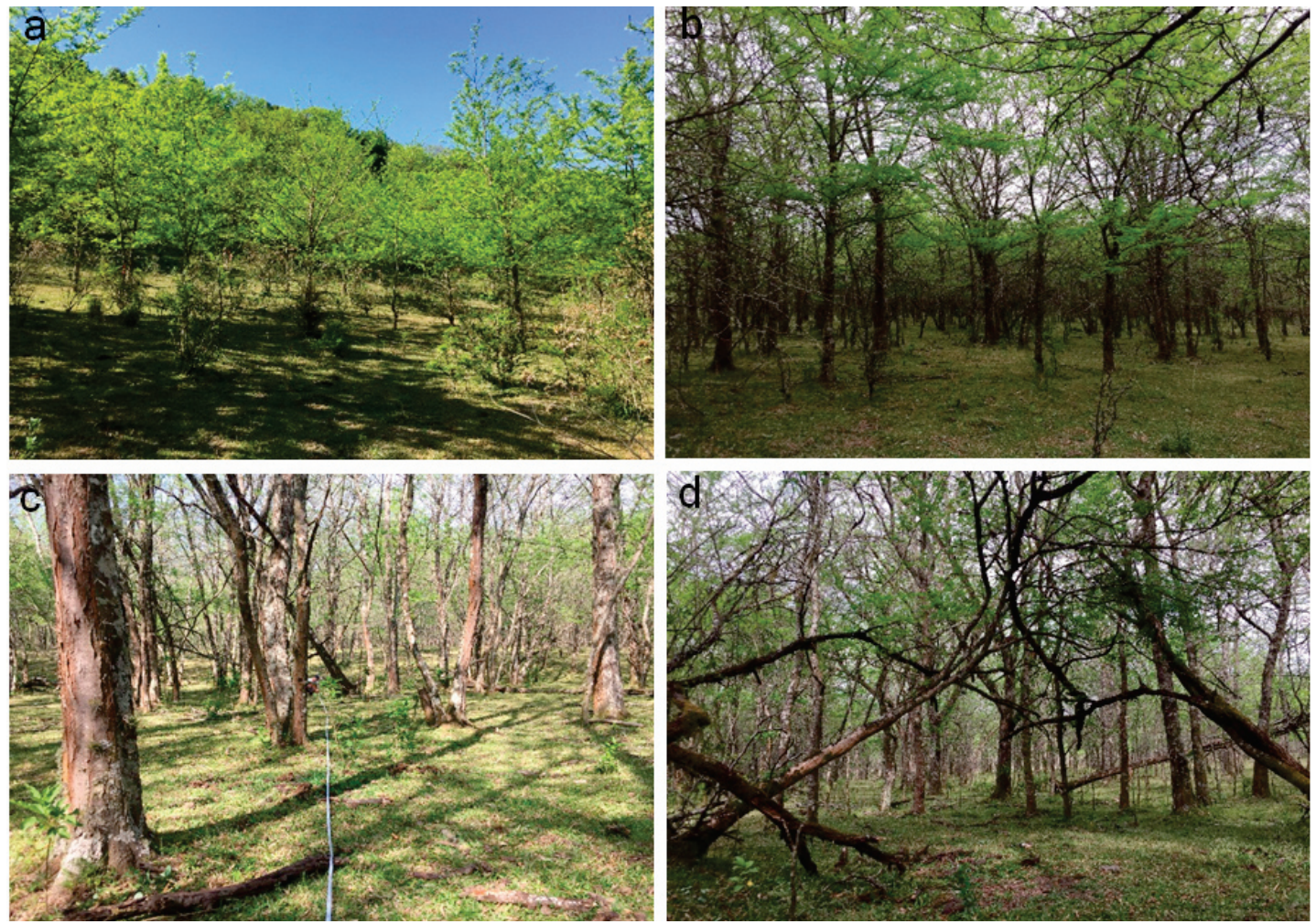

Figura 2. Fotografías de las parcelas de Gleditsia triacanthos de 9 (a), 18 (b), 25-29 (c) y 36-37 (d) años, ubicadas en el valle de La Sala (Tucumán). Se observa que a los 9 años el bosque tiene baja altura (a), a los 18 años el bosque tiene mayor densidad (b), a los 25-29 años se destacan árboles de mayor diámetro (c), y a los 36-37 años aumentan los árboles caídos e inclinados (d). Las fotografías fueron tomadas por José Tisone.

Figure 2. Photos of the Gleditsia triacanthos plots of 9 (a), 18 (b), 25-29 (c), and 36-37 (d) years, established in La Sala Valley (Tucumán). The 9-years-old forests have low height (a); at 18-years, forests have more density (b); at 25-29 years, have trees with higher diameter (c); and at 36-37 years, forests have more fallen trees (d). Photos belong to José Tisone.

Tabla 2. Resultados de los modelos empleados para comparar variables estructurales de las parcelas por edad. Los valores de $P$ corresponden a comparaciones a posteriori entre las parcelas. Las diferencias significativas están señaladas con un asterisco.

Table 2. Results of the models that compare structural variables of the plots per age. $P$ values results from post-hoc comparisons. Asterisks indicate significant differences.

\begin{tabular}{llccc}
\hline Variable & & Parcela de 9 años & Parcela de 18 años & Parcela de 25-29 años \\
\hline \multirow{2}{*}{ Densidad de tallos } & Parcela de 18 años & $<0.0001^{*}$ & & \\
& Parcela de 25-29 años & $0.0001^{*}$ & $0.0095^{*}$ & \\
& Parcela de 36-37 años & $<0.0001^{*}$ & 0.0571 & 0.9275 \\
DAP promedio & Parcela de 18 años & $<0.0001^{*}$ & & \\
& Parcela de 25-29 años & $<0.0001^{*}$ & $<0.0001^{*}$ & 0.5546 \\
\multirow{2}{*}{ Altura promedio } & Parcela de 36-37 años & $<0.0001^{*}$ & $<0.0001^{*}$ & \\
& Parcela de 18 años & $<0.0001^{*}$ & & \\
& Parcela de 25-29 años & $<0.0001^{*}$ & $0.0047^{*}$ & 0.8901 \\
\hline
\end{tabular}

años. Considerando los árboles $\geq 5 \mathrm{~cm}$ de diámetro a la altura del pecho, en el bosque de 25-29 años se registró una mayor riqueza que en bosques más jóvenes. Además de $G$. triacanthos y $V$. aroma, se registraron a las especies X. pubescens y M. alba.
Bosque de 36-37 años

Estebosque tuvolas siguientes características: 1) presencia de árboles caídos, muertos o en mal estado, con mayor frecuencia que en bosques más jóvenes (Figura 2), 2) individuos arbóreos de G. triacanthos con menos espinas 
y menor densidad de tallos secundarios, y 3) incremento de la riqueza de especies de árboles (Figura 1), pero con una densidad mucho menor que la de G. triacanthos. El DAP y la altura media de los árboles no difirieron significativamente del bosque de 25-29 años (Tabla 2). Se registraron 8 especies de árboles con un diámetro $\geq 5 \mathrm{~cm}$, aunque del total de tallos / ha (783) sólo 27 corresponden a especies distintas a $G$. triacanthos. Se registraron las especies nativas A. edulis (10 tallos/ha), Azara salicifolia (2 tallos/ha), Celtis ehrenbergiana (2 tallos/ha), Cestrum lorentzianum (2 tallos/ha), Condalia buxifolia (4 tallos/ha), X. pubescens (4 tallos/ha) y la exótica M. alba (2 tallos/ha). Como en los bosques de 18 y 25-29 años, registramos renovales de especies nativas creciendo en la proximidad de los árboles de $G$. triacanthos y protegidos por sus espinas ( $A$. edulis, B. salicifolius, C. ehrenbergiana, Eugenia uniflora y $X$. pubescens). No registramos individuos adultos ni renovales de V. aroma.

\section{DisCUSIÓN}

Con el establecimiento de una cronosecuencia (parcelas de diferentes edades), analizamos la dinámica de la invasión de G. triacanthos en el valle de La Sala (Tucumán). La dinámica de los bosques dominados por esta especie pudo caracterizarse considerando un rango temporal desde los 9 hasta los 37 años, que es la edad de los bosques secundarios más viejos del área. En una primera etapa, la invasión de G. triacanthos fue similar a la reportada para otras regiones del país (i.e., estuvo facilitada por la dispersión de sus vainas por el ganado y por su comportamiento típico de especie pionera, es decir es una de las primeras especies colonizadoras) (Grau and Aragón 2000; Mazia et al. 2001; Ruiz Selmo et al. 2007). Su invasión en campos abandonados fue relativamente rápida debido a que a los 9 años ya encontramos un bosque bajo donde G. triacanthos es la especie arbórea dominante. Sin embargo, en este bosque aún encontramos claros o espacios abiertos posiblemente mantenidos por el ganado (que limita el establecimiento y desarrollo de las plántulas de G. triacanthos; Capó et al. 2016; Sosa et al. 2018) y que son colonizados en años posteriores.

En esta primera etapa, $V$. aroma fue la única especie arbórea nativa que registramos en los bosques invadidos, aunque con una densidad más baja que la de G. triacanthos. Vachellia aroma es también una especie pionera pero por su baja densidad en estos bosques posiblemente tenga desventajas competitivas frente a G. triacanthos. Por ejemplo, en un estudio realizado en el Chaco Serrano de Córdoba, G. triacanthos tuvo mayor densidad de frutos, semillas y plántulas alrededor de los árboles adultos que $V$. aroma (Ferreras et al. 2014), que aventajan a la especie invasora para colonizar los campos abandonados. En etapas avanzadas de la invasión, G. triacanthos alcanza mayor densidad, altura y diámetro, posiblemente disminuyendo el acceso a la luz a $V$. aroma, que es una especie de baja altura y de lugares abiertos. Es probable que por este motivo encontramos muchos tallos de $V$. aroma en mal estado en la parcela de 18 años dominada por G. triacanthos, y ningún adulto ni recluta de $V$. aroma en la parcela de 36-37 años.

En etapas avanzadas de la invasión, luego de 18 años de establecido el bosque, aumentó la riqueza de especies de árboles (considerando renovales y adultos), aunque con una densidad muy baja en comparación con la de G. triacanthos. Todas estas especies de árboles se caracterizaron por tener frutos carnosos y por crecer siempre en la proximidad de los árboles de G. triacanthos. Esta particular disposición espacial pudo deberse, en parte, a la dispersión de los frutos carnosos por parte de las aves frugívoras que habitan estos bosques y que utilizan las ramas de G. triacanthos como perchas (Moreno Ruiz Holgado 2012). Además, es posible que el ganado haya contribuido a esta disposición de los renovales, que son ramoneados en áreas abiertas y tienen mayor supervivencia cuando crecen cercanos a los árboles de G. triacanthos que los protegen con sus espinas.

En el área, G. triacanthos dominó bosques secundarios de diferentes edades por su alta densidad, lo cual probablemente dificulta su reemplazo. Considerando las parcelas establecidas, en los bosques de 9 a 37 años de edad, más del 90\% de los tallos fueron de G. triacanthos. Si bien algunas especies nativas pueden colonizar parches invadidos por G. triacanthos (de Viana and Colombo Speroni 2000), la probabilidad de reemplazar a la especie invasora es muy baja teniendo en cuenta la información obtenida en las parcelas. Suponemos que Vachellia aroma, que es una colonizadora temprana, fue eliminada por ser competitivamente inferior a la invasora, y si bien otras especies arbóreas nativas pudieron establecerse en etapas avanzadas, su densidad fue muy baja. En base a estos resultados, 
pudimos observar que la regeneración natural de la composición nativa en el área es poco probable debido a que los árboles nativos no reemplazan a G. triacanthos en el tiempo. Los resultados obtenidos en este trabajo podrían ser útiles para brindar recomendaciones para restaurar bosques invadidos por G. triacanthos: 1) la restauración debe ser activa, dado que la especie invasora domina distintas etapas sucesionales y la regeneración de especies arbóreas nativas es escasa, 2) se debe controlar a G. triacanthos, principalmente a aquellos individuos que están compitiendo con árboles nativos plantados o establecidos de forma espontánea, y 3) se deben plantar árboles nativos que de adultos superen en altura a $G$. triacanthos y que toleren la sombra.

\section{CONCLUSIÓN}

En este trabajo describimos la estructura y la composición de los bosques de G. triacanthos en el valle de La Sala (Tucumán), e inferimos su dinámica temporal a través de una cronosecuencia de parches de bosque de 9, 18, 25-29 y 36-37 años donde esta especie es dominante. Los bosques de G. triacanthos presentan una dinámica típica de un rodal en sucesión: establecimiento temprano como especie pionera, incremento del tamaño y la altura de los árboles con el paso del tiempo, y presencia deárboles caídos, inclinados o en mal estado en etapas avanzadas. En estos bosques, G. triacanthos continúa siendo dominante con el paso del tiempo y el reemplazo por otras especies de árboles nativos es poco probable debido a las habilidades competitivas que presenta como invasora.

Agradecimientos. Queremos agradecer a J. Tisone, A. Gregorio y F. Escobar, por su ayuda en el establecimiento de las parcelas. Agradecemos a E. Araoz y J. Carilla por enseñarnos dendrocronología y ayudarnos a tomar las muestras. Este trabajo fue financiado por el proyecto PUE0023.

\section{REFERENCIAS}

Bustos, M. 1991. Variación de la diversidad de micromamíferos a través de un gradiente sucesional secundario de la selva montana. Tesis de grado. Universidad Nacional de Tucumán, Tucumán, Argentina. Pp. 98.

Caballero, N. 2013. Análisis de las invasiones especies leñosas exóticas en las Quebradas del Norte de Uruguay. Tesis de grado. Universidad de la República, Uruguay. Pp. 66.

Capó, E. A., R. Aguilar, and D. Renison. 2016. Livestock reduces juvenile tree growth of alien invasive species with a minimal effect on natives: a field experiment using exclosures. Biological Invasions 18:2943-2950. https://doi.org/ $10.1007 /$ s10530-016-1185-3.

Csurhes, S. M., and D. Kriticos. 1994. Gleditsia triacanthos L. (Caesalpiniaceae), another thorny, exotic fodder tree gone wild. Plant Protection 9:101-105.

De Viana, M. L., and F. Colombo Speroni. 2000. Invasión de Gleditsia triacanthos L. (Fabaceae) en el bosque de San Lorenzo, Salta, Argentina. Pp. 71-84 en H. R. Grau and R. Aragón (eds.). Ecología de árboles exóticos en las Yungas argentinas. LIEY-UNT, Tucumán, Argentina.

Di Marzio, W., M. E. Sáenz, J. Alberdi, N. Fortunato, M. Tangorra, V. Capello, M. Tortorelli, and G. Ambrini. 2009. Estrategia de manejo de acacia negra (Gleditsia triacanthos) en la cuenca del río Luján. Evaluación ecotoxicológica del herbicida Togar BT. Revista Argentina de Ecotoxicología y Contaminación Ambiental 1:1-7.

Faggi, A. M., K. Krellenberg, R. Castro, M. Arriaga, and W. Endlicher. 2006. Biodiversity in the Argentinean rolling pampa ecoregion: changes caused by agriculture and urbanization. Erdunke 60:127-138. https://doi.org/10.3112/ erdkunde.2006.02.04.

Fernández, R. D., S. J. Ceballos, A. Malizia, and R. Aragón. 2017. Gleditsia triacanthos (Fabaceae) in Argentina: a review of its invasion. Australian Journal of Botany 65(3):203-213. https://doi.org/10.1071/BT16147.

Ferreras, A. E., G. Funes, and L. Galetto. 2014. Evaluación interanual de las estrategias regenerativas de la especie exótica invasora Gleditsia triacanthos en relación a la nativa Acacia aroma en el bosque chaqueño serrano de Córdoba (Argentina). Bosque 35:155-162. https:/ / doi.org/10.4067/S0717-92002014000200003.

Ghersa, C. M., and R. León. 2001. Ecología del Paisaje Pampeano: consideraciones para su manejo y conservación. Pp. 471-512 en Z. Naveh and A. S. Lieberman (eds.). Ecología de Paisajes, Teorías y Aplicaciones. Editorial Facultad de Agronomía de la UBA, Buenos Aires, Argentina.

Giorgis, M. A., and P. A. Tecco. 2014. Árboles y arbustos invasores de la provincia de Córdoba (Argentina): una contribución a la sistematización de bases de datos globales. Boletín de la Sociedad Argentina de Botánica 49(4): 581-603. https://doi.org/10.31055/1851.2372.v49.n4.9991.

Grau, H. R., and R. Aragón. 2000. Arboles invasores de la Sierra de San Javier, Tucumán, Argentina. Pp. 5-20 en H. R. Grau and R. Aragón (eds.). Ecología de árboles exóticos en las Yungas argentinas. LIEY-UNT, Tucumán, Argentina.

Grau, H. R., L. Paolini, A. Malizia, and J. Carilla. 2010. Distribución, estructura y dinámica de los bosques de la sierra de San Javier (Tucumán, Argentina). Pp. 33-50 en H. R. Grau (ed.). Ecología de una interfase natural-urbana. La sierra de San Javier y el Gran San Miguel de Tucumán. EDUNT, Tucumán, Argentina.

IPBES. 2020. Página web https://ipbes.net/glossary?initial=1), visitada el 11/06/2020. 
Marco, D. E., and S. A. Páez. 2000. Invasion of Gleditsia triacanthos in Lithraea ternifolia montane forest of Central Argentina. Environmental Management 26:409-419. https://doi.org/10.1007/s002670010098.

Mazia, C., E. Chaneton, C. Ghersa, and R. León R. 2001. Limits to tree species invasion in pampean grassland and forest plant communities. Oecologia 128:594-602. https://doi.org/10.1007/s004420100709.

Mazia, C. N., E. J. Chaneton, M. Machera, A. Uchitel, M. V. Feler, and C. M. Ghersa. 2010. Antagonistic effects of large- and small-scale disturbances on exotic tree invasion in a native tussock grassland relict. Biological Invasions 12:3109-3122. https://doi.org/10.1007/s10530-010-9702-2.

Moreno Ruiz Holgado, M. M. 2012. Comparación de diversidad de aves en bosques nativos de ribera y bosques de Gleditsia triacanthos de la cuenca media del río San Javier. Tesis de grado. Universidad Nacional de Tucumán, Tucumán, Argentina. Pp.38.

Osinaga-Acosta, O., S. Báez, F. Cuesta, J. Carilla, A. Malizia, L. R. Malizia, H. R. Grau, A. D. Brown and T. Lomáscolo. 2014. Módulo 1, Monitoreo de especies arbóreas. Pp. 18-60 en O. Osinaga-Acosta, S. Báez, F. Cuesta, A. Malizia, J. Carilla, N. Aguirre and L. Malizia (eds.). Monitoreo de diversidad vegetal y carbón en bosques andinos - Protocolo extendido. CONSESAN, Lima, Perú.

Prieto, A. R., A. M. Blasi, C. G. De Francesco, and C. Fernández. 2004. Environmental history since 11,000 14C yr B.P. of the northeastern Pampas, Argentina, from alluvial sequences of the Luján River. Quaternary Research 62:146-161. https://doi.org/10.1016/j.yqres.2004.04.006.

Quiroga, P. A., and J. P. Juliá. 2011. Estudio de una cuenca de río subtropical de montaña: pautas para su gestión ecosistémica. Pp. 159-174 en H. R. Fernández and H. Barber (eds.). La Cuenca del Río Lules: Una aproximación multidisciplinaria a su complejidad. EDUNT, Tucumán, Argentina.

R Core Team. 2020. R: A language and environment for statistical computing. R Foundation for Statistical Computing, Vienna, Austria. http:/ /www.R-project.org.

Richardson, D. M., and M. Rejmánek. 2011. Trees and shrubs as invasive alien species-a global review. Diversity and Distributions 17:788-809. https://doi.org/10.1111/j.1472-4642.2011.00782.x.

Ruiz Selmo, F. E., P. G. Minotti, A. Scopel, and M. Parinbelli. 2007. Análisis de la heterogeneidad fisonómico-estructural de la vegetación del Parque Nacional El Palmar y su relación con la invasión por leñosas exóticas. Pp. 257-263 en R. Rivas, A. Grisotto and M. Sacido (eds.). Teledetección - Hacia un mejor entendimiento de la dinámica global y regional. Martin, Buenos Aires, Argentina.

Sosa, B., D. Romero, K. Mello, L. Profumo, C. Chiale, and M. Achkar. 2017. Bases ecológicas en el control de Gleditsia triacanthos en el Parque Nacional Esteros de Farrapos e Islas del Río Uruguay. Pp. 50-53 en A. Brazeiro (ed.). Recientes avances en investigación para la gestión y conservación del bosque nativo de Uruguay. UDELAR, Montevideo, Uruguay.

Tognetti, P. M., N. Mazia, and G. Ibáñez. 2019. Seed local adaptation and seedling plasticity account for Gleditsia triacanthos tree invasion across biomes. Annals of Botany 124(2):307-318. https://doi.org/10.1093/aob/mcz077.

Zalba, S. M., and C. B. Villamil. 2002. Woody plant invasion in relictual grasslands. Biological Invasions 4:55-72. https: //doi.org/10.1023/A:1020532609792. 